\title{
Electronic Structure and Spectroscopic Studies of the Molecule ScS
}

\author{
Rawad Halabi, Mahmoud Korek \\ Faculty of Science, Beirut Arab University, Beirut, Lebanon \\ Email: fkorek@yahoo.com
}

Received 22 February 2015; accepted 7 April 2015; published 9 April 2015

Copyright (C) 2015 by authors and Scientific Research Publishing Inc.

This work is licensed under the Creative Commons Attribution International License (CC BY).

http://creativecommons.org/licenses/by/4.0/

(c) () Open Access

\begin{abstract}
Theoretical investigation of the lowest electronic states of ScS molecule, in the representation ${ }^{2 s+1} \Lambda^{(+/-)}$, has been performed via CASSCF and MRCI (single and double excitations without Davidson correction) calculations. The calculated potential energy curves (PECs), permanent dipole moment curves (PDMCs), and spectroscopic constants are reported for the 10 lowest electronic states. The eigenvalues $E_{v}$, the rotational constants $B_{v}$, and the centrifugal distortion constants $D_{v}$ have been calculated for various vibrational levels. The comparison of the present results with the available experimental data in literature shows an overall good agreement. To the best of our knowledge, 6 electronic states of the ScS molecule, between $11600 \mathrm{~cm}^{-1}$ and $15000 \mathrm{~cm}^{-1}$ are not yet observed experimentally and are investigated in the present work for the first time.
\end{abstract}

\section{Keywords}

Ab Initio Calculation, Electronic Structure, Spectroscopic Constants, Potential Energy Curves, Dipole Moments, Vibration-Rotation Calculation

\section{Introduction}

The molecules containing transition metal are species of chemical and astrophysical importance. Scandium is the first transition metal atom which has only one d-electron in the ground state. Diatomic molecules like ScS are simple transition metal-containing systems in which d-electrons take part in bonding and provide ideal models for understanding the electronic structure and reactivity (chemiluminescent reactions).

To the best of our knowledge, the experimental work for the ScSmolecule is that of Gengler et al. [1]-[3]. They studied the $\mathrm{A}^{2} \Pi-\mathrm{X}^{2} \Sigma^{+}$and $\mathrm{B}^{2} \Pi-\mathrm{X}^{2} \Sigma^{+}$band system of scandium monosulfide, ScS, using Fourier transform emission spectroscopy (FT) and laser excitation spectroscopy (LIF). The only theoretical work for ScS in literature is that of Bauschlicher and Langhoff [4] for the ground $\mathrm{X}^{2} \Sigma^{+}$and $(1)^{2} \Pi$ states using $a b$ initio method.

In the present work, $a b$ initio investigations of the lowest lying electronic states of the ScS molecule have 
been performed via CAS-SCF/MRCI (Complete Active Space Self Consistent Field, Multireference Configuration Interaction) method. Multireference CI calculations (single and double excitations without Davidson corrections) in which the entire CAS-SCF configuration space is used as the reference, are performed to account the correlation effects. The potential energy curves (PECs), the transition dipole moment curves (TDMCs) together with the energy minimum for the ground state $T_{e}$, the equilibrium internuclear distance $R_{e}$, the harmonic frequency $\omega_{\mathrm{e}}$ and the rotational constant $\mathrm{B}_{\mathrm{e}}$ have been obtained for the 10 lowest-lying electronic states. Six electronic states have been investigated in the present work for the first time.

\section{Method of Calculations}

In the present work, $a b$ initio investigations of the lowest-lying electronic states of the ScS molecule have been performed via CASSCF method. Multireference CI calculations (single and double excitations without Davidson corrections) were performed to determine the correlation effects. The entire CASSCF configuration space was used as a reference in the MRCI calculation which have been performed via the computational chemistry program MOLPRO [5]. The scandium species is treated in an all electron scheme [6] [7], the 21 electrons of the scandium atom are considered using the contracted Gaussian basis set used by Bauschlicher and Langhoff [4] for $\mathrm{s}$, $\mathrm{d}$, and $\mathrm{f}$ functions then we have taken $\mathrm{p}$ function from 6 - $31 \mathrm{G}^{* *}$ basis set [5] which has 16 diffused functions contracted set of Gaussian functions. The sulfur species is treated as a system with 16 inner electrons taken into account the pseudopotential $\mathrm{W}^{\mathrm{ps}}$ [8]-[10] together with the corresponding Gaussian basis set. In the range of the internuclear distance $\mathrm{R}_{\mathrm{e}}$ around equilibrium distances of its ground state, the ScS molecule is assumed to be mainly ionic as many transition-metals $\mathrm{Sc}^{+} \mathrm{S}^{-}$. The potential energy calculations for the states ${ }^{2 \mathrm{~s}^{+1}} \Lambda^{(+/-)}$of the molecule ScS have been carried using a CASSCF method. Among the 37 electrons explicitly considered for ScS (21 electrons for Sc and 16 for S) 27 inner electrons were frozen in subsequent calculations so that 10 valence electrons were explicitly treated. The active space contains $4 \sigma$ (Sc: $\left.4 \mathrm{~s}, 3 \mathrm{~d}_{0}\right), 1 \pi\left(3 \mathrm{~d}_{ \pm 1}\right)$ and $1 \delta\left(\right.$ Sc: $\left.3 \mathrm{~d}_{ \pm 2}\right)$, this corresponds to 6 active molecular orbitals orbitals in the $\mathrm{C}_{2 \mathrm{v}}$ symmetry distributed into irreducible representation in the following way: $3 \mathrm{a}_{1}, 1 \mathrm{~b}_{1}, 1 \mathrm{~b}_{2}, 1 \mathrm{a}_{2}$, noted [3111]. The doubly occupied orbitals $2 \sigma\left(\mathrm{S}: 5 \mathrm{~s}, 5 \mathrm{p}_{0}\right)$, and $1 \pi\left(\mathrm{S}: 5 \mathrm{p}_{ \pm 1}\right)$ of sulfur have been considered as inactive in the CASSCF calculations. Correlation effects for the ten valence electrons have been taken into account through multireference calculations MRCI (single and double excitations without Davidson correction) where the entire CASSCF configuration space has been used as reference. Calculations have been performed via the computational program MOLPRO [5] taking advantage of the graphical user interface GABEDIT [11].

\section{Results and Discussion}

Calculations have been performed for 51 internuclear distances in the range $2.00 \AA$ - $3.02 \AA$ for 10 states in therepresentation ${ }^{2 \mathrm{~s}+1} \Lambda^{(+-)}$. The potential energy curves (PECs) for the states ${ }^{2,4} \Sigma,{ }^{2,4} \Pi,{ }^{2,4} \Delta$ and ${ }^{2,4} \Phi$ in the considered range of Rare drawn respectively in Figure 1, Figure 2. For each state, transition energies with respect to the minimum energy of the ground state $T_{e}$, equilibrium internuclear distance $R_{e}$, harmonic frequency $\omega_{e}$ and the rotational constant $B_{e}$ have been calculated. These constants are displayed in Table 1 together with the literature values from both theoretical and experimental investigations.

The comparison of our results with the values for $T_{e}$ calculated by different techniques in the literature for the 10 lowest-lying doublet and quartet electronic states shows that, 7 values are in good agreement where the relative difference is $1.00 \%\left[{ }^{[3}\right.$ Ref.1] $\leq \delta \mathrm{T}_{\mathrm{e}} / \mathrm{T}_{\mathrm{e}} \leq 4.70 \%\left[{ }^{\mathrm{c1-2}} \mathrm{Ref} .1\right]$ for the states $\mathrm{X}^{2} \Sigma^{+},(2)^{2} \Sigma^{+}$and $(1)^{2} \Pi$ and one value for the $(1)^{2} \Pi$ state calculated in Ref. [1] by the SDCI method shows a larger relative difference equal to $13.0 \%$. For $\omega_{\mathrm{e}}$ the comparison of our calculated values with the 16 values given in the literature for the 10 lowest electronic states showed an overall good agreement where the relative difference is $0.80 \%\left[{ }^{c 5}\right.$ Ref.1 for $\left.(1)^{2} \Pi\right]$ $\leq \delta \omega_{\mathrm{e}} / \omega_{\mathrm{e}} \leq 12.3 \%\left[{ }^{\mathrm{c} 4} \mathrm{Ref} .1\right.$ for $\left.(2)^{2} \Sigma^{+}\right]$. Two larger values are obtained for the state (1) ${ }^{2} \Delta$ calculated in Ref. [1] by the CPF and SDCI $+\mathrm{R}$ methods. Since the value of $\omega_{\mathrm{e}}$ depends on the shape of the potential energy curves near the minimum, this difference may be explained by the shape of the investigated potential curve by this method. The values of $R_{e}$ and $B_{e}$ obtained in literature are in excellent agreement with our calculated values with relative differences $0.3 \%$ [ ${ }^{\mathrm{c} 2}$ Ref.1] $\leq \delta \mathrm{R}_{\mathrm{e}} / \mathrm{R}_{\mathrm{e}} \leq 2.1 \%$ [ ${ }^{\mathrm{c} 1-1}$ Ref.1] and $1.6 \%\left[{ }^{\mathrm{b} 3}\right.$ Ref. 4$] \leq \delta \mathrm{B}_{\mathrm{e}} / \mathrm{B}_{\mathrm{e}} \leq 4.1 \%$ [ ${ }^{\mathrm{b} 1}$ Ref.2] respectively for the states $\mathrm{X}^{2} \Sigma^{+},(2)^{2} \Sigma^{+}$and $(1)^{2} \Delta$ while the relative differences become larger for the state $(1)^{2} \Pi$.

By using the canonical functions approach Korek et al. [12]-[19] and the cubic spline interpolation between each two consecutive points of the potential energy curves obtained from the $a b$ initio calculation of the ScS 


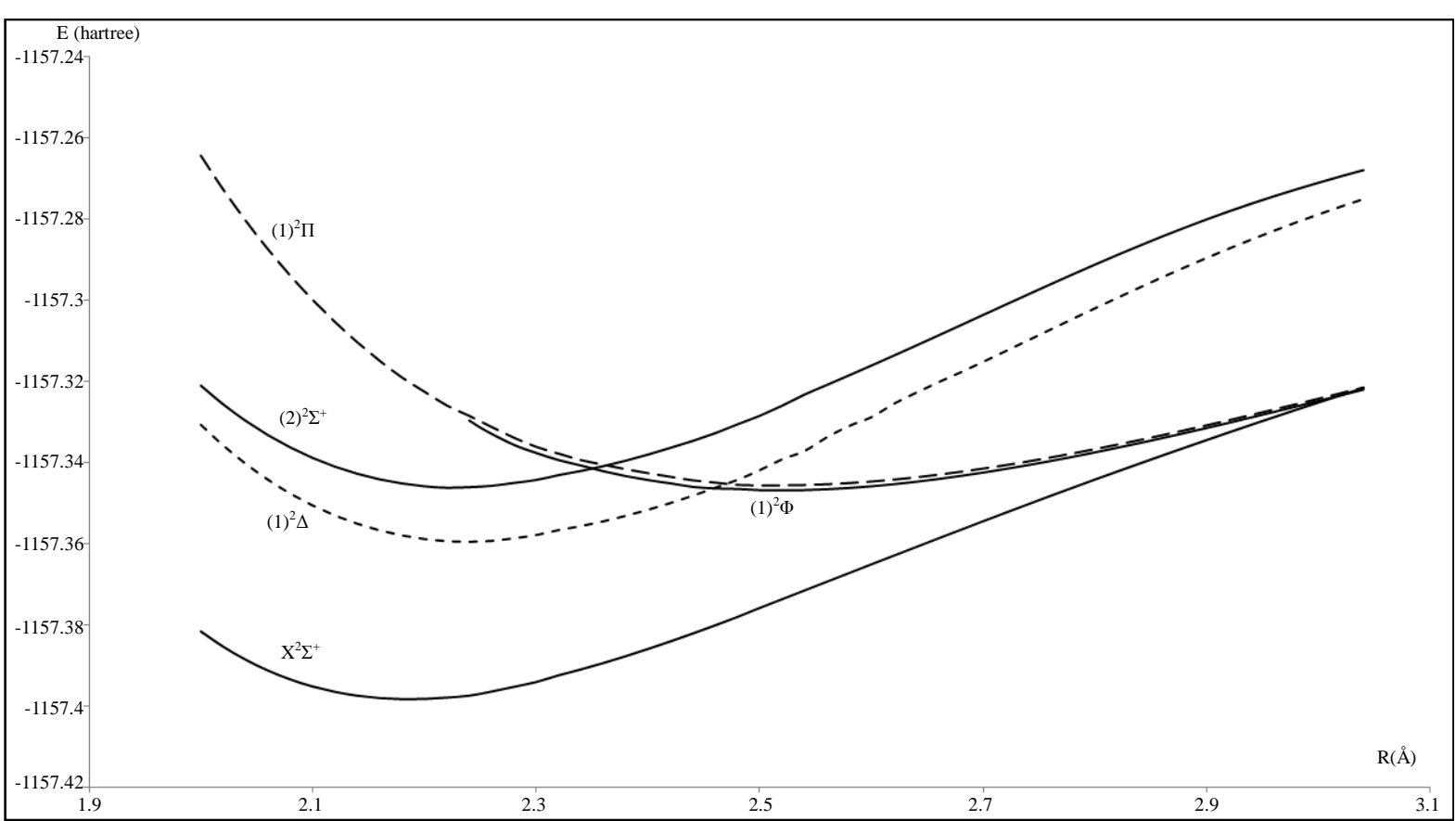

Figure 1. Potential energy curves of the electronic states $X^{2} \Sigma,(1)^{2} \Delta,(2)^{2} \Sigma,(1)^{2} \Pi$ and $(1)^{2} \Phi$ of the molecule ScS.

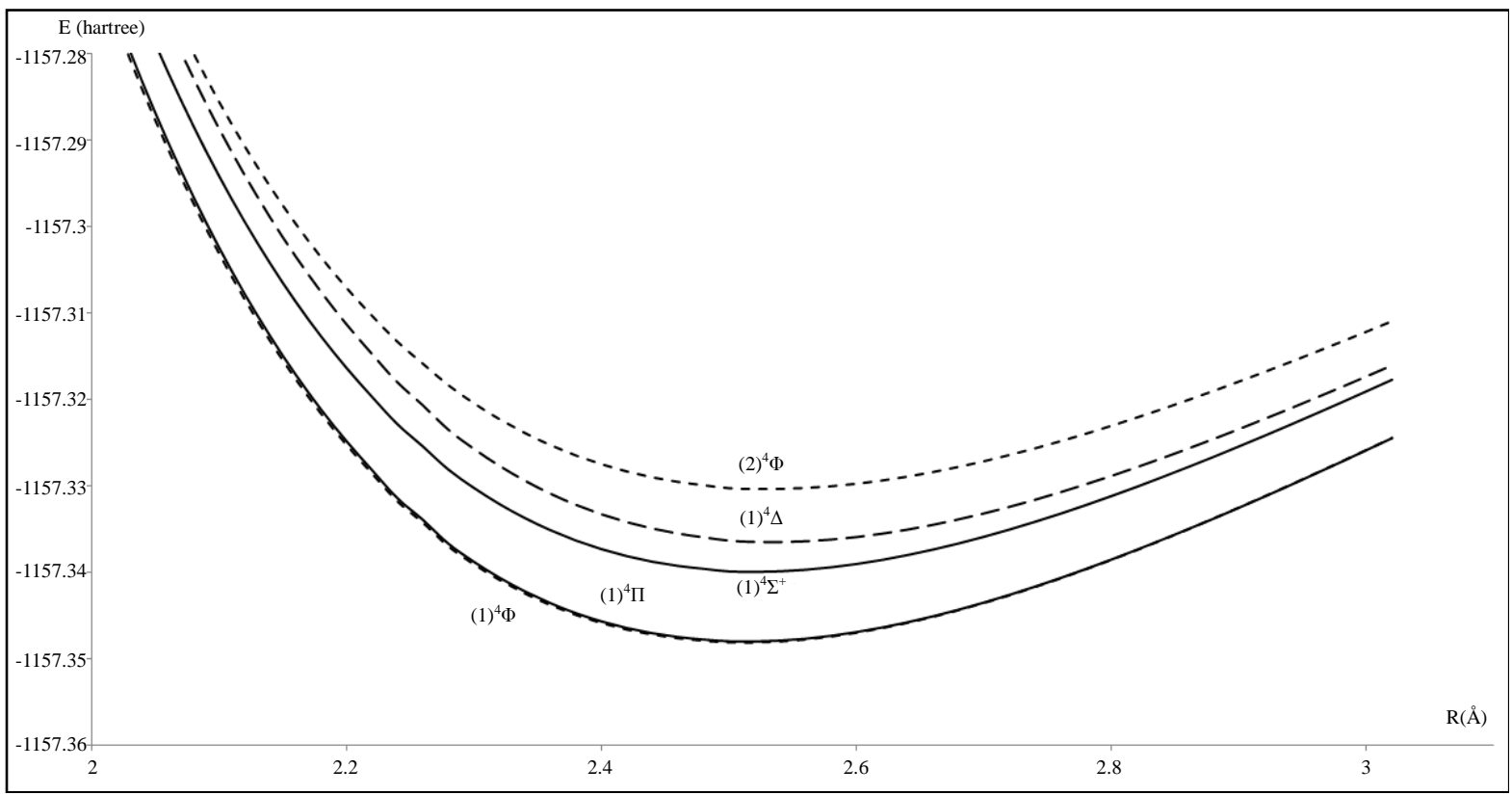

Figure 2. Potential energy curves of the electronic states $(1)^{4} \Sigma^{+},(1)^{4} \Delta,(1)^{4} \Phi,(1)^{4} \Pi$ and $(2)^{4} \Phi$ of the molecule ScS.

molecule, the eigenvalue $E_{v}$, the rotational constant $B_{v}$, the centrifugal distortion constants $D_{v}$, The abscissas of the turning point $\mathrm{R}_{\min }$ and $\mathrm{R}_{\max }$ have been calculated for the considered electronic states up to the vibrational levels $\mathrm{v}=10$. These values for the states $(1)^{4} \Sigma,(1)^{4} \Delta,(1)^{4} \Phi,(2)^{4} \Pi$ and $(2)^{4} \Phi$ (as illustration) are given in Table 2 . The absence of the comparison with other results is because of the calculation of these values here for the first time. Then, using the canonical functions approach, the eigenvalues $E_{v}$, the rotational constants $B_{v}$ and the centrifugal distortion constants $D_{\mathrm{v}}$ are calculated for different vibrational levels $\mathrm{v}$ for the seven molecular states of ScS $\left((1)^{2} \Pi,(2)^{2} \Sigma,(1)^{4} \Sigma,(1)^{4} \Delta,(1)^{4} \Phi,(2)^{4} \Pi\right.$ and $\left.(2)^{4} \Phi\right)$. These results are displayed in Table 2, respectively. The comparison of our calculated values with those obtained experimentally for the rotational constants $B_{v}$ and 
Table 1. Calculated spectroscopic constants for the lowest 10 electronic states of ScS.

\begin{tabular}{|c|c|c|c|c|}
\hline States & $\mathrm{T}_{\mathrm{e}}\left(\mathrm{cm}^{-1}\right)$ & $\mathrm{R}_{\mathrm{e}}(\hat{\AA})$ & $\omega_{\mathrm{e}}\left(\mathrm{cm}^{-1}\right)$ & $\mathrm{B}_{\mathrm{e}} \times 10^{2}\left(\mathrm{~cm}^{-1}\right)$ \\
\hline \multirow{9}{*}{$\mathrm{X}^{2} \Sigma^{+}$} & $0.0^{\mathrm{a}}$ & $2.181^{\mathrm{a}}$ & $540.6^{\mathrm{a}}$ & $18.929^{\mathrm{a}}$ \\
\hline & $0.0^{\mathrm{b}}$ & $2.1375^{\mathrm{b}-1}$ & & $19.7294^{\mathrm{b}-1}$ \\
\hline & & $2.144^{\mathrm{b}-2}$ & & $19.61^{\mathrm{b}-2}$ \\
\hline & & $2.139^{\mathrm{b}-3}$ & & $19.71^{\mathrm{b}-3}$ \\
\hline & $0.0^{\mathrm{c}}$ & $2.1354^{c-1-1}$ & $565.2^{\mathrm{c}-1}$ & \\
\hline & & $2.167^{c-2}$ & $518^{\mathrm{c}-2}$ & \\
\hline & & $2.177^{\mathrm{c}-3}$ & $503^{c-3}$ & \\
\hline & & $2.126^{c-4}$ & $607^{c-4}$ & \\
\hline & & $2.125^{c-5}$ & $608^{c-5}$ & \\
\hline \multirow{5}{*}{$(1)^{2} \Delta$} & & $2.229^{\mathrm{a}}$ & $581.6^{\mathrm{a}}$ & $18.126^{\mathrm{a}}$ \\
\hline & & $2.236^{c-2}$ & $486^{c-2}$ & \\
\hline & $8760^{\mathrm{a}}$ & $2.237^{\mathrm{c}-3}$ & $488^{\mathrm{c}-3}$ & \\
\hline & & $2.221^{c-4}$ & $513^{c-4}$ & \\
\hline & & $2.221^{\mathrm{c}-5}$ & $515^{c-5}$ & \\
\hline$(1)^{4} \Phi$ & 11031 & 2.513 & 353.5 & 14.265 \\
\hline$(1)^{4} \Pi$ & 11058 & 2.515 & 353.1 & 14.247 \\
\hline$(1)^{2} \Phi$ & 11332 & 2.528 & 360.0 & 14.002 \\
\hline \multirow{5}{*}{$(2)^{2} \Sigma^{+}$} & $11604^{\mathrm{a}}$ & $2.2223^{\mathrm{a}}$ & $557.4^{\mathrm{a}}$ & $18.240^{\mathrm{a}}$ \\
\hline & $12456^{\mathrm{b}-1}$ & $2.20391^{\mathrm{b}-1} 2.2056^{\mathrm{b}-3}$ & $488.737^{\mathrm{b}-1}$ & $18.5583^{\mathrm{b}-1}$ \\
\hline & $12456^{\mathrm{b}-3}$ & $2.159^{c-3}$ & & $18.53^{\mathrm{b}-3}$ \\
\hline & & $2.143^{c-5}$ & $567^{c-3}$ & \\
\hline & & & $582^{c-5}$ & \\
\hline \multirow{7}{*}{$(1)^{2} \Pi$} & $11538.4^{\mathrm{a}}$ & $2.497^{\mathrm{a}}$ & $494.9^{\mathrm{a}}$ & $14.444^{\mathrm{a}}$ \\
\hline & $11090.4^{\mathrm{b}-1}$ & $2.2079^{b-1}$ & & $18.4910^{\mathrm{b}-1}$ \\
\hline & $11000^{\mathrm{c}-1-2}$ & & & \\
\hline & $11691^{\mathrm{c}-2}$ & $2.236^{\mathrm{c}-2}$ & $468^{\mathrm{c}-2}$ & \\
\hline & $11659^{\mathrm{c}-3}$ & $2.236^{\mathrm{c}-3}$ & $470^{\mathrm{c}-3}$ & \\
\hline & $10698^{\mathrm{c}-4}$ & $2.218^{c-4}$ & $488^{c-4}$ & \\
\hline & $10032^{\mathrm{c}-5}$ & $2.22^{\mathrm{c}-5}$ & $491^{\mathrm{c}-5}$ & \\
\hline$(1)^{4} \Sigma$ & 12828 & 2.522 & 350.6 & 14.158 \\
\hline$(1)^{4} \Delta$ & 13581 & 2.537 & 344.9 & 13.995 \\
\hline$(2)^{4} \Phi$ & 14928 & 2.533 & 333.8 & 14.043 \\
\hline
\end{tabular}

${ }^{\text {a }}$ Present Theoretical study; ${ }^{b}$ Ref. [1]-[3]; ${ }^{(\mathrm{b}-1)}$ Ref. [1]; ${ }^{(\mathrm{b}-2)}$ Ref. [2]; ${ }^{(\mathrm{b}-3)}$ Ref. [3]; ${ }^{\mathrm{c}}$ Ref. [4]. 
Table 2. Values of the eigenvalues $E_{v}$, the abscissa of the turning points $R_{\min }, R_{\max }$ the rotational constants $B_{v}$ and the centrifugal distortion constants $D_{v}$ for thedifferent vibrational levels of $(1)^{2} \Pi,(2)^{2} \Sigma^{+},(1)^{4} \Sigma^{+},(1)^{4} \Delta,(1)^{4} \Phi,(2)^{4} \Pi$, and (2) $\Phi$ electronic states of the ScS molecule.

\begin{tabular}{|c|c|c|c|c|c|c|}
\hline & $\mathrm{v}$ & $\mathrm{E}_{\mathrm{v}}\left(\mathrm{cm}^{-1}\right)$ & $\mathrm{R}_{\min }(\AA)$ & $\mathrm{R}_{\max }(\AA)$ & $\mathrm{B}_{\mathrm{v}} \times 10^{2}\left(\mathrm{~cm}^{-1}\right)$ & $\mathrm{D}_{\mathrm{v}} \times 10^{8}\left(\mathrm{~cm}^{-1}\right)$ \\
\hline \multirow{4}{*}{$(1)^{2} \Pi$} & 0 & 173.4 & 1.786 & 2.593 & $\begin{array}{l}14.17^{\mathrm{a}} \\
18.45^{\mathrm{b}}\end{array}$ & $\begin{array}{c}9.35^{\mathrm{a}} \\
10.67^{\mathrm{b}}\end{array}$ \\
\hline & 1 & 521.2 & 1.791 & 2.649 & $\begin{array}{l}14.15^{\mathrm{a}} \\
18.36^{\mathrm{b}}\end{array}$ & $\begin{array}{c}8.66^{\mathrm{a}} \\
10.83^{\mathrm{b}}\end{array}$ \\
\hline & 2 & 872.9 & 1.795 & 2.691 & 14.04 & 8.73 \\
\hline & 3 & 1224.1 & 1.799 & 2.728 & 14.00 & 10.63 \\
\hline \multirow{2}{*}{$(2)^{2} \Sigma^{+}$} & 0 & 282.9 & 2.171 & 2.277 & $\begin{array}{l}18.16^{\mathrm{a}} \\
18.56^{\mathrm{b}}\end{array}$ & $\begin{array}{c}7.96^{\mathrm{a}} \\
10.82^{\mathrm{b}}\end{array}$ \\
\hline & 1 & 826.9 & 2.133 & 2.337 & $\begin{array}{l}18.02^{\mathrm{a}} \\
18.46^{\mathrm{b}}\end{array}$ & $\begin{array}{c}9.45^{\mathrm{a}} \\
10.88^{\mathrm{b}}\end{array}$ \\
\hline \multirow{10}{*}{$(1)^{4} \Sigma^{+}$} & 0 & 178.1 & 2.453 & 2.596 & 14.13 & 9.33 \\
\hline & 1 & 525.1 & 2.405 & 2.655 & 14.08 & 9.52 \\
\hline & 2 & 868.3 & 2.374 & 2.698 & 14.01 & 9.60 \\
\hline & 3 & 1208.3 & 2.349 & 2.734 & 13.95 & 9.28 \\
\hline & 4 & 1547.0 & 2.329 & 2.767 & 13.87 & 9.53 \\
\hline & 5 & 1883.1 & 2.312 & 2.797 & 13.82 & 9.74 \\
\hline & 6 & 2216.1 & 2.296 & 2.825 & 13.74 & 8.57 \\
\hline & 7 & 2549.0 & 2.282 & 2.852 & 13.67 & \\
\hline & 8 & 2877.8 & 2.271 & 2.877 & & \\
\hline & 9 & 3532.0 & 2.247 & 2.926 & & \\
\hline \multirow{11}{*}{$(1)^{4} \Phi$} & 0 & 176.9 & 2.467 & 2.612 & 14.24 & 9.26 \\
\hline & 1 & 529.4 & 2.417 & 2.674 & 14.19 & 9.50 \\
\hline & 2 & 877.6 & 2.385 & 2.718 & 14.12 & 9.27 \\
\hline & 3 & 1224.2 & 2.361 & 2.796 & 14.05 & 9.36 \\
\hline & 4 & 1568.5 & 2.339 & 2.789 & 13.99 & 9.42 \\
\hline & 5 & 1910.3 & 2.321 & 2.821 & 13.92 & 9.04 \\
\hline & 6 & 2250.6 & 2.305 & 2.849 & 13.85 & 9.21 \\
\hline & 7 & 2588.8 & 2.291 & 2.878 & 13.78 & 8.80 \\
\hline & 8 & 2925.6 & 2.277 & 2.904 & 13.69 & \\
\hline & 9 & 3261.6 & 2.267 & 2.931 & 11.10 & \\
\hline & 10 & 3595.5 & 2.256 & 2.955 & 13.00 & \\
\hline
\end{tabular}


R. Halabi, M. Korek

\section{Continued}

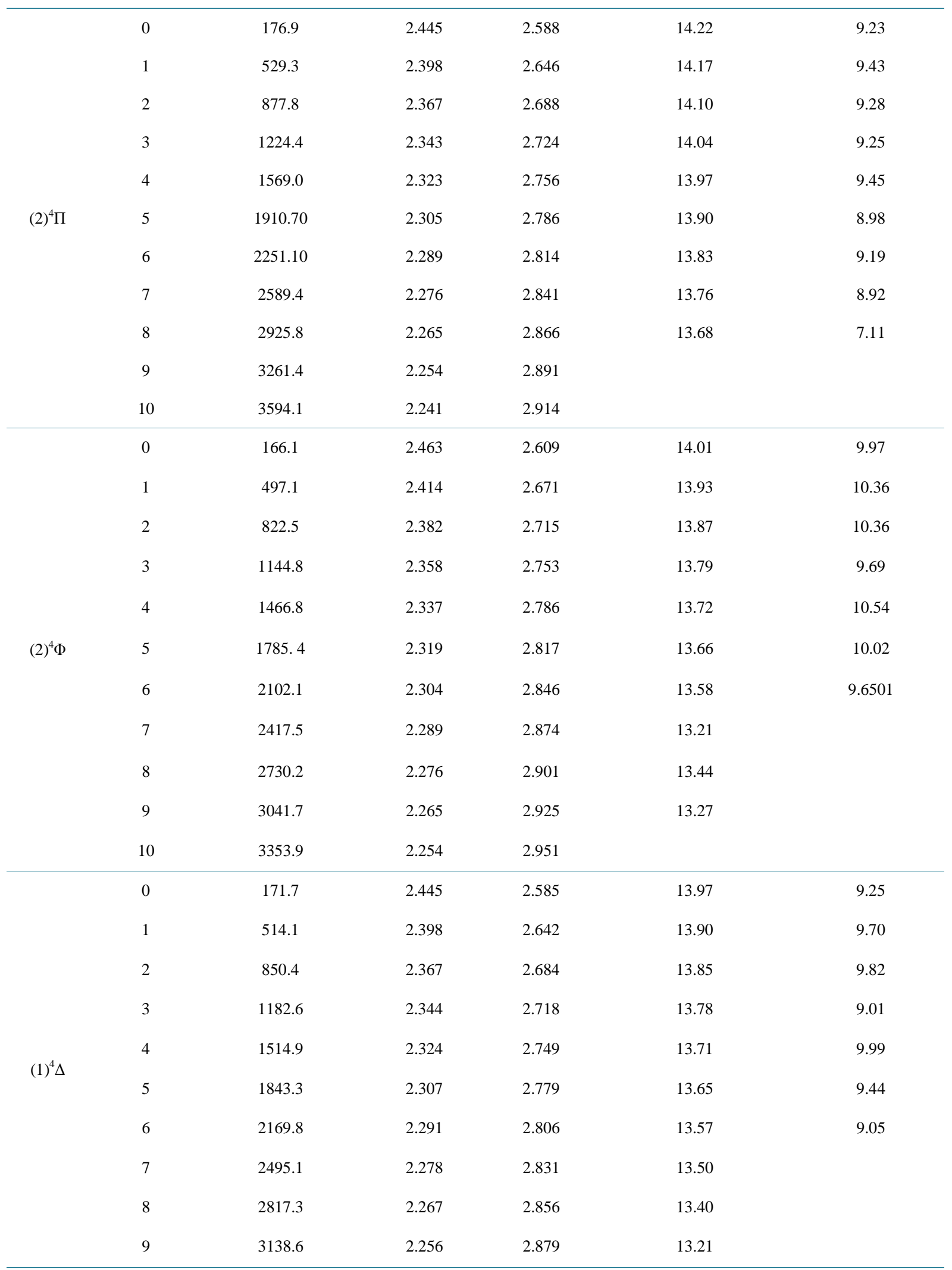

${ }^{\mathrm{a}}$ Present work; ${ }^{\mathrm{b}}$ Ref. [3]. 
the centrifugal distortion constants $\mathrm{D}_{\mathrm{v}}$ showed a good agreement for the state $(2)^{2} \Sigma$ with averaged relative differences $\delta \mathrm{B}_{\mathrm{v}} / \mathrm{B}_{\mathrm{v}}=2.1 \%$ and $\delta \mathrm{D}_{\mathrm{v}} / \mathrm{D}_{\mathrm{v}}=35.8 \%$ for $\mathrm{v}=0$, and these differences are $\delta \mathrm{B}_{\mathrm{v}} / \mathrm{B}_{\mathrm{v}}=2.4 \%$ and $\delta \mathrm{D}_{\mathrm{v}} / \mathrm{D}_{\mathrm{v}}=$ $15.1 \%$ for $\mathrm{v}=1$. This agreement becomes less for the state $(1)^{2} \Pi$, with averaged relative differences $\delta \mathrm{B}_{\mathrm{v}} / \mathrm{B}_{\mathrm{v}}=$ $23.2 \%$ and $\delta \mathrm{D}_{\mathrm{v}} / \mathrm{D}_{\mathrm{v}}=14.1 \%$ for $\mathrm{v}=0$, and these differences are $\delta \mathrm{B}_{\mathrm{v}} / \mathrm{B}_{\mathrm{v}}=22.9 \%$ and $\delta \mathrm{D}_{\mathrm{v}} / \mathrm{D}_{\mathrm{v}}=25.1 \%$ for $\mathrm{v}=1$.

\section{Conclusion}

In the present work, the $a b$ initio investigation for 10 low-lying electronic states of the ScS molecule has been performed via CASSCF/MRCI method. The potential energy and the dipole moment curves have been determined along with the spectroscopic constants $T_{e}, R_{e}, \omega_{e}$ and the rotational constant $B_{e}$ for these states. The comparison of our results with those obtained theoretically in literature showed a good accuracy. By using the canonical functions approach [12]-[19], the eigenvalue $E_{v}$, the rotational constant $B_{v}$, the centrifugal distortion constants $D_{v}$, and the abscissas of the turning points $R_{\min }$ and $R_{\max }$ were calculated up to the vibrational level $v=$ 10. Six electronic states have been investigated in the present work for the first time.

\section{References}

[1] Gengler, J., Chen, J.H., Steimle, T.C., Ram, R.S. and Bernath, P.F. (2006) Journal of Molecular Spectroscopy, 237, 36-45. http://dx.doi.org/10.1016/j.jms.2006.02.006

[2] Steimle, T.C., Marr, A.J. and Goodridge, D.M. (1997) Journal of Chemical Physics, 107, 10406-10414. http://dx.doi.org/10.1063/1.474204

[3] Fenot, B., Femenias, J.L. and Stringat, R. (1979) Journal of Molecular Spectroscopy, 78, 40-50. http://dx.doi.org/10.1016/0022-2852(79)90034-1

[4] Bauschlicher Jr., C.W. and Langhoff, S.R. (1986) Journal of Chemical Physics, 85, 5936. http://dx.doi.org/10.1063/1.451505

[5] Werner, H.J., Knowles, P.J., Lindh, R., Manby, F.R., Schütz, M., Celani, P., Korona, T., Rauhut, G., Amos, R.D., Bernhardsson, A., Berning, A., Cooper, D.L., Deegan, M.J.O., Dobbyn, A.J., Eckert, F., Hampel, C., Hetzer, G., Lloyd, A.W., McNicholas, S.J., Meyer, W., Mura, M.E., Nicklass, A., Palmieri, P., Pitzer, R., Schumann, U., Stoll, H., Stone, A.J., Tarroni, R. and Thorsteinsson, T. MOLPRO Is a Package of Ab-Initio Programs.

[6] Chong, D.P., Langhoff, S.R., Bauschlicher, C.W., Walch, S.P. and Partridge, H. (1986) Journal of Chemical Physics, 85, 2850-2860. http://dx.doi.org/10.1063/1.451044

[7] Langhoff, S.R., Pettersson, L.G.M., Bauschlicher, C.W. and Partridge, H. (1987) Journal of Chemical Physics, 87, 481. http://dx.doi.org/10.1007/s00214-006-0168-3

[8] Dunning Jr., T.H. and Hay, P.J. (1985) Journal of Chemical Physics, 82, 2664. http://dx.doi.org/10.1063/1.448263

[9] Hurley, M.M., Pacios, L.F., Christiansen, P.A., Ross, R.B. and Ermler, W.C. (1986) Journal of Chemical Physics, 84, 6840. http://dx.doi.org/10.1063/1.453069

[10] La John, L.A., Christiansen, P.A., Ross, R.B., Atashroo, T. and Ermler, W.C. (1987) Journal of Chemical Physics, 87, 2812. http://dx.doi.org/10.1063/1.450689

[11] Allouche, A.R. (2011) Journal of Computational Chemistry, 32, 174-182. http://dx.doi.org/10.1002/jcc.21600

[12] Kobeissi, H. and Korek, M. (1982) International Journal of Quantum Chemistry, 22, 23-29. http://dx.doi.org/10.1002/qua.560220104

[13] Kobeissi, H. and Korek, M. (1994) Journal of Physics B: Atomic, Molecular and Optical Physics, 27, 3653. http://dx.doi.org/10.1088/0953-4075/27/16/012

[14] Kobeissi, H., Korek, M., Dagher, M. and Chaalan, A. (1983) Journal of Computational Chemistry, 4, 218-225. http://dx.doi.org/10.1002/jcc.540040212

[15] Kobeissi, H. and Korek, M. (1985) Journal of Physics B: Atomic, Molecular and Optical Physics, 18, 1115. http://dx.doi.org/10.1088/0022-3700/18/6/016

[16] Korek, M. and Kobeissi, H. (1993) Canadian Journal of Chemistry, 71, 313-317. http://dx.doi.org/10.1139/v93-046

[17] Korek, M. and Kobeissi, H. (1992) Journal of Computational Chemistry, 13, 1103-1108. http://dx.doi.org/10.1002/jcc.540130909

[18] Korek, M. (1999) Computer Physics Communications, 119, 169-178. http://dx.doi.org/10.1016/S0010-4655(98)00180-5

[19] Kobeissi, H., Korek, M. and Dagher, M. (1989) Journal of Molecular Spectroscopy, 138, 1-12. http://dx.doi.org/10.1016/0022-2852(89)90092-1 